\title{
Rationale of a Cohort Study on Risk of Obstetrical Outcomes Associated with Iron Supplementation during Pregnancy
}

\author{
Andy Muela Mbangama, Barthélémy Tandu-Umba, Roger Mwimba Mbungu \\ Department of Obstetrics and Gynecology, University Clinics of Kinshasa, Kinshasa, DR Congo \\ Email: dymuela@yahoo.fr
}

How to cite this paper: Mbangama, A.M., Tandu-Umba, B. and Mbungu, R.M. (2018) Rationale of a Cohort Study on Risk of Obstetrical Outcomes Associated with Iron Supplementation during Pregnancy. Open Journal of Obstetrics and Gynecology, 8, 598-609.

https://doi.org/10.4236/ojog.2018.86066

Received: April 6, 2018

Accepted: June 10, 2018

Published: June 13, 2018

Copyright $\odot 2018$ by authors and Scientific Research Publishing Inc. This work is licensed under the Creative Commons Attribution International License (CC BY 4.0).

http://creativecommons.org/licenses/by/4.0/

cc) (i) Open Access

\begin{abstract}
Background: Anemia is one of the most widely prevalent disorders, affecting the lives of almost half a billion women of reproductive age, contributing to over 100,000 maternal and almost 600,000 perinatal deaths (mostly through pre-term delivery, low birth weight) each year. Increased risk of infant mortality and reduced cognitive development and reduced energy levels which affect productivity in adults are cited. During pregnancy increased requirements, inadequate intake of iron and other micronutrients and parasitic (malaria, hookworm) as wells as bacterial (mostly urinary tract) infections are the main causes. In order to reduce such maternal and neonatal burden, it has been worldwide admitted to adopt cost-effective preventive interventions during pregnancy, including iron-folic acid supplementation, de-hookworming medication and anti-malarial prevention or treatment. Intestinal absorption of iron is limited by a lot of factors including bioavailability, iron status of the woman, substances accompanying or contained in diet, chelating agents such as diet fibers or calcium salts. Any supplementation put additional constraint in terms of absorption. Unabsorbed iron is known to have pro-oxidant properties likely to induce production of free radicals. These in turn might induce oxidative stress accountable for in generation of many obstetrical outcomes. This potential link between oxidative stress resulting from free radicals hyperproduction induced by non absorbed iron and harmful maternal/perinatal conditions is rarely questioned by searchers. Objectives: To determine overall (food and supplemented) iron consumption, iron and oxidative status in a cohort of pregnant women and to seek associations between findings and adverse obstetrical outcomes. Methods: At the University Clinics of Kinshasa, we designed a protocol for a prospective cohort study dealing with clinical and biochemical parameters of oxidative stress among pregnant women iron supplemented. Women with a single pregnancy not exceeding 19
\end{abstract}


weeks without obvious pathology, regardless of age and parity, were eligible for inclusion in the study. Conclusion: This study is expected to assess consequences of oral iron supplementation during pregnancy in terms of obstetrical outcomes associated with oxidative stress linked to unabsorbed iron.

\section{Keywords}

Obstetrical Outcomes, Iron Supplementation, Oxidative Stress

\section{Introduction}

Anemia is defined as a hemoglobin value below the lower limits of its normal range, resulting in tissue hypoxia, the consequences of which will be reflected in a variety of symptoms such as fatigue, lightheadedness, weakness, and exertion dyspnea [1] [2]. World Health Organization (WHO) estimated the prevalence of anemia in developed and developing countries among pregnant women at $14 \%$ and $51 \%$, respectively [3]. In the Democratic Republic of Congo, particularly at University Clinics of Kinshasa, the frequency of anemia during pregnancy is estimated at 53.4\% [4]. Causes include iron deficiency as a leading one, parasitic (malaria, hookworms) and bacterial infections (mostly urinary tract infection), micronutrient deficiencies including folic acid, vitamin $\mathrm{A}$, and vitamin B12, and genetically inherited hemoglobinopathies such as drepanocytosis. All these causes are most prevalent in women of reproductive age and children, mostly in developing areas facing lower energy and iron intake [5].

Despite conflicting findings from reviews of observational studies and randomized trials, abundant literature is consistent with strong association between prenatal anemia and high risk of maternal and perinatal morbidity/mortality (through pre-term delivery and low birth weight). Increased risk of infant mortality and reduced cognitive development are cited in children and reduced energy levels which affect productivity in adults [6]. Fear of such a burden has urged the WHO to adopt cost-effective preventive interventions during pregnancy, including iron-folic acid supplementation, de-hookworming medication and anti-malarial prevention or treatment [7].

Recommended nutritional iron intake is $16 \mathrm{mg} /$ day for women [8]. Irrespective of its origin (animal, vegetal, or oral supplement), ingested iron is absorbed in the upper part of small intestine [9] [10], the rate depending on various factors such as: origin (iron of vegetal origin being less bioavailable than animal), patient's reserves (the less the reserves, the more the absorption), salt type (ferrous salts absorbed three times faster than ferric) and preparation type (enteric coatings and long-acting products transporting iron after duodenum and proximal jejunum likely to reduce absorption) [9] [11] [12]. So, of 12 - $18 \mathrm{mg}$ of recommended iron intake, only $1-2 \mathrm{mg}$ is expected to be absorbed. Additional limitation to iron absorption is due to certain pathologies (intestinal parasitic and urinary tract infections) and chelating substances such as phytates, phos- 
phates, tannins, fibers and calcium salts [13] [14] [15]. All these limiting conditions are common in developing countries also known to be at great need for oral supplementation during pregnancy.

Unabsorbed iron is known to have pro-oxidant properties likely to induce production of free radical oxygen species [16] [17] [18] [19]. Indeed, in presence of free iron, oxygenated intermediates catalyze conversion of hydrogen peroxide into hydroxyl radicals by Fenton reaction: $\mathrm{H}_{2} \mathrm{O}_{2}+\mathrm{Fe}^{2+} \rightarrow \mathrm{OH}^{-}+\mathrm{OH}+\mathrm{Fe}^{3+}$ [20] [21], the products of which attack cell membranes, proteins, lipids, carbohydrates and DNA [22]. Alteration and thereafter rupture of membranes of cells' organelles (mitochondria, lysosomes and sarcoplasm) lead to cell death [23] [24].

During pregnancy cellular damage associated with oxidative stress is known to result in TNF- $\alpha$ production that activates leukocytes as they pass through intervillous chamber. These activated leukocytes release stress mediators that are basis for pluri-visceral damages, alteration of sub-decidual angiogenesis, reduction in intrauterine blood flow and mother-fetus transfer of stress substances, resulting in myometrial irritability and fetal inflammatory climate [25]. These effects are accountable for in reportedly high frequencies of pregnancy loss [26], shortening of gestational age, prematurity, restriction of birth weight [27]-[33] and neonatal intravascular hemorrhage [34] [35]. Furthermore, Oxidative radicals or their metabolites interfere with various protective functions of vascular endothelium: vasodilation, barrier function, anti-aggregant activity [36] [37] [38] [39] [40]. Disruption of this process of body signaling vasodilatation results in increase of maternal blood pressure, which is likely to lead not only to preeclampsia-eclampsia (PE) but also to intrauterine growth restriction (IUGR) and premature labor [41].

As of link between oxidative stress and diabetes during pregnancy, it is known to result from radical hyperproduction associated with hyperglycemia and antioxidant's defenses decrease [42]. This can lead to gestational diabetes mellitus (GDM). Moreover, during diabetic pathology, hyperglycemia constitutes an oxidative stress, responsible for increase in glycolysis which, by increasing mitochondrial membrane potential, increases formation of free radicals [43] [44].

So, supplementation during pregnancy might contribute to huge unabsorbed amount of iron which in turn might induce oxidative stress accountable for in generation of many obstetrical adverse outcomes, most of them being also persistently cited as associated with maternal anemic situation [4]. However, the potential link between oxidative stress resulting from free radicals hyperproduction induced by iron supplementation and harmful maternal/perinatal conditions is rarely questioned by searchers. This is the main objective of our study.

\section{Objectives}

In order to assess potential links between free radicals hyperproduction induced by maternal iron supplementation and adverse pregnancy outcomes in our setting this study aims to determine overall (food and supplemented) iron con- 
sumption, iron and oxidative status in a cohort of pregnant women and to seek associations between findings and adverse obstetrical outcomes.

\subsection{Rationale for the Study}

It is to question safety of recommended oral iron supplementation during pregnancy (120 - $300 \mathrm{mg}$ per day). As compared with the quantity to be absorbed (only $1-2 \mathrm{mg}$ ), the research is based on potential oxidative stress induced by non absorbed iron that remains in contact with intestinal mucosa (=Fenton reaction). Due to importance of factors limiting iron absorption in developing areas it is postulated that in orally supplemented pregnant women huge unabsorbed iron (either preventive or curative) might be harmful at some degree.

\subsection{Design and Methods of the Study}

\subsubsection{Overview of the Study}

This protocol was designed to test hypothesis that in iron supplemented pregnant women unabsorbed free fraction is associated with occurrence of adverse obstetrical outcomes. Considering 53.4\% (the prevalence of anemia in pregnant women of our setting), the minimum size of sample is 54, calculated according to the formula $n \geq \frac{2\left(Z_{\alpha}+Z_{1-\beta}\right)^{2} p(1-p)}{\left(p_{0}-p_{1}\right)^{2}}$ [45]. In this formula,

- $p$ is the anemia prevalence in our country (53.4\%) and $p=\frac{p_{0}+p_{1}}{2}$;

- $Z_{\alpha}$ is the confidence coefficient; it's the value of $Z_{1-\frac{\alpha}{2}}$ for a bilateral test (1.96);

- $Z_{1-\beta}$ corresponds to the power of the test $(1-\beta)$; the latter is the probability of finding a significant difference (1.645);

- $\alpha$ is the error risk of type I (0.05);

- $p_{\mathrm{o}}$ is the expected proportion of pregnant women who have not received a martial supplementation and who will develop adverse obstetrical outcomes;

- $p_{1}$ is the expected proportion of pregnant women who have received a martial supplementation and who will develop adverse obstetrical outcomes; this $p_{1}$ proportion is estimated by the investigator according to the estimated $p_{0}$ value and the importance of the difference between $p_{\mathrm{o}}$ and $p_{1}$ that he thinks possible and desirable to highlight;

- Considering that $p_{1}=R R \times p_{0} \quad($ with $\mathrm{RR}=2)$ and $p=\frac{p_{0}+p_{1}}{2} \rightarrow$ $0.534=\frac{p_{0}+\left(2 p_{0}\right)}{2}$.

So $p_{0}=0.36$ et $p_{1}=0.7$.

After incorporating these elements in the formula, our minimal sample size was calculated as 54 pregnant women. So, taking into account non-responses or unsatisfactory data and the prenatal frequency of consultations at University Clinics in Kinshasa, we majored our sample to 74 pregnant women to be fol- 
lowed for antenatal care until delivery. This study has been designed and financed by our own funds.

\subsubsection{Patients' Selection}

\section{Inclusion criteria}

1) Single pregnancy of not more than 19 weeks (starting point of martial supplementation), regardless of age and parity.

2) Absence of any pathology likely to have a negative influence on pregnancy (hypertension, diabetes mellitus, sickle cell disease, HIV, malaria, respiratory insufficiency).

3) Informed and written consent to participate in the study.

Exclusion criteria:

1) Pregnancy that has evolved into an abortion;

2) Free decision to discontinue participation in the study at any time.

The sampling was carried out consecutively for all pregnant women presenting themselves at prenatal care, taking into account inclusion and exclusion criteria.

\subsubsection{Variables of the Study and Subjects Monitoring}

1) Socio-demographic and anthropometric variables: age, marital status, parity, weight, height, number and abortion type, education level, economic status, body mass index (BMI) calculated from weight and height. The information about the socio-demographic and anthropometric variables, and Medico-surgical and gynaecological history was obtained during the pregnant women anamnesis conducted at prenanal care.

2) Medico-surgical and gynecological history: diabetes mellitus or gestational diabetes mellitus, hypertension, pre-eclampsia, eclampsia, congenital malformation, fetal macrosomia, low birth weight, stillbirth, polyhydramnios, prematurity, premature rupture of membranes, caesarean section.

3) Medical conditions known to limit iron absorption, such as intestinal parasitic infection.

4) Pregnancy parameters: gestational age (at recruitment, onset of martial supplementation and delivery), amount of iron supplemented and ingested, pregnancy pathologies, delivery parameters (mode of delivery, APGAR, birth weight and birth length).

5) Nutritional iron intake measured through a 24 -hour recall using a questionnaire of common foods, including stuffs and habits likely to impede or boost iron absorption such as pica, chelating substances such as phytates, phosphates, tannins, fibers, alcohol and tobacco.

6) Supplemental iron intake determined with a sheet containing details on the salt type (ferrous or ferric), and duration of the supplementation.

7) Biological parameters: iron status (hemoglobin, hematocrit, ferritin, serum iron, transferrin and iron saturation capacity), oxidative status including enzyme antioxidants (superoxide dismutase or SOD, uric acid) and biological markers of oxidative stress effects (oxidized LDL, ferritin, blood glucose) and direct exami- 
nation of stool. Hemoglobin and hematocrit to be measured using a KX-21N automated hematology analyzer. Serum iron (Fe), uric acid and fasting blood glucose to be measured with a manual spectrophotometrical assay (kit Spectrum 722, 18TS1505007). Transferrin, iron saturation capacity of transferrin and superoxide dismutase to be measured with a manual spectrophotometrical assay (kit Biolabo Diagnostic's, Kenza Max Biochemistry serial n ${ }^{\circ} 34840$ ). Ferritin and oxidized LDL to be analyzed by the ELISA method (on the Elisa analyzer, $\mathrm{Hu}$ mareader signe plus 1800). Direct examination of stool with binocular microscope Olympus XSZ-107BN.

8) Adverse obstetrical outcomes in terms of preterm labor and premature labor ( $<37$ weeks of gestation), pre-eclampsia (PE), GDM (to be diagnosed between weeks 26 and 28 of gestation using a glucose tolerance test), premature rupture of membranes (PRM) and IUGR (based on local charts as a birth weight less than the $10^{\text {th }}$ percentile for the estational age group) and low birth weight (birth weight $<2500 \mathrm{~g}$ ), stillbirth (defined as death of a fetus after 28 weeks of gestation), perinatal mortality (deaths including stillbirths and neonatal deaths before 7 days of life).

\subsection{Proceeding}

The study is to be conducted during 10 months (September 2017 throughout June 2018) at the department of Gynecology and Obstetrics, University Clinics of Kinshasa, DR Congo. One of us (MMA) has been recruiting patients of the study among pregnant women of not more than 19 weeks (starting point of martial supplementation) regardless of age and parity. After checking for pathologic conditions (arterial hypertension, diabetes mellitus, sickle cell disease, HIV, malaria, respiratory insufficiency) for exclusion if noted, blood, stool and urine samples are collected, in addition to maternal anthropometrics, and basic demographic information. Anemia is defined as hemoglobin $<10 \mathrm{~g} / \mathrm{L}$ or hematocrit $<30 \%$. Iron status determined using ferritin concentrations (normal value defined as serum ferritin $<12 \mu \mathrm{g} / \mathrm{L}$ ). Oxidative status is assessed using plasma conserved at $-20^{\circ} \mathrm{C}$ until analysis. The same samplings and assessments are done at 28 gestation week and at term. Obstetrical complications are managed as usually. All the pregnant women included in the study are to be followed at antenatal care by the principal investigator. Women with anemia $(\mathrm{Hb}<10 \mathrm{~g} \%)$ at recruitment start iron supplementation for curative purposes $(320 \mathrm{mg} /$ day of ferric ammonium citrate) before the 19th week, while the others receive preventive iron supplementation ( $160 \mathrm{mg} /$ day of ferric ammonium citrate) by the 19th week. Iron is associated with folate (15 mg/day).

\subsection{Expected Study Outcomes}

Within this study we aim to determine iron status of the pregnant in our environment; the iron amount absorbed after oral supplementation and to identify insufficient absorption determinants; the oxidative status of pregnant woman 
before and after oral iron supplementation; the correlation between amount of unabsorbed iron, iron status, oxidative status and adverse obstetrical outcomes; and the determinants of oxidative stress and adverse obstetrical outcomes. The duration of the supplementation for each pregnant woman is to be determined at the end of the study.

\subsection{Statistical Considerations}

Data will be entered using Microsoft Excel 2007 software and exported to SPSS 21.0 for analysis. For normally distributed parametric data, comparisons of averages are to be made using t-test or ANOVA and comparison of proportions with chi-square test.

Correlation between amount of unabsorbed iron, iron status, oxidative status and adverse pregnancy outcomes is planned. Odds ratio will be used to measure strength of association between risk factors and amount of unabsorbed iron on the one hand and oxidative stress and adverse obstetrical outcomes on the other. Multivariate logistic regression analysis will identify independent determinants of oxidative stress and adverse obstetrical outcomes. Testing will be stated significant at $p \leq 0.05$.

\subsection{Ethical Considerations}

This project was agreed by Ethics Committee of Public Health School of University of Kinshasa. Let us note that sampling takes place within usual framework of pregnancy monitoring. For all pregnant women included in the study, informed and signed is obtained in advance. For treatment purposes, their identity and telephone numbers serve to locate them later on if necessary. To guarantee confidentiality, this information is filled on a coupon kept under saddle by the principal investigator alone. No further reference is made to this coupon except for purpose of treatment for anemia or obstetric complications.

\section{Discussion}

Antenatal anemia is most prevalent in low income countries where it is responsible for maternal and perinatal morbidity/mortality (through pre-term delivery and low birth weight) [46]. Increased risk of infant mortality and reduced cognitive development are cited in children and reduced energy levels which affect productivity in adults [47]. Fear of such a burden has urged the World Health Organization to adopt cost-effective preventive interventions during pregnancy including iron-folic acid supplementation, de-hookworming medication and anti-malarial prevention or treatment [7].

So, improvement in hemoglobin and iron status of mothers is expected to reduce the risk of these adverse outcomes. However, evidence of such a link fails to be found in all studies dealing with prenatal iron supplementation. This could be owed too many confounding factors such as lack to take in account baseline iron status of pregnant women and ignorance of enhancing or limiting factors of iron 
absorption. This is particularly important in developing countries also known to be at great need for oral supplementation during pregnancy and where limiting conditions of iron absorption are very common [48]. Our study aims to collect findings related to this aspect.

Furthermore, there has been abundant literature admitting close relationships between maternal oxidative stress and body or serum iron status [17] [18] [19], meaning that iron supplementation might bring a risk for both mother and infant, which makes it difficult to delineate the mutual part of anemia and supplemented iron in occurrence of obstetrical outcomes. Besides, although current literature obviously relates this potential risk to absorbed iron our study aims to question also unabsorbed iron. Additionally, we find it necessary to consider the dosage recommended by WHO for routine daily prenatal iron supplementation (100 - $300 \mathrm{mg}$ in our setting).

Another matter of concern relates to the fact that pro-oxidant properties of iron have been most described as accountable for in generation of gestational diabetes mellitus or restriction of fetal growth [49]. So, other obstetrical outcomes seem to have been neglected.

All these aspects will be addressed in our study.

\subsection{The Strengths of the Study}

1) Although it seems obvious that there is a potential link between oxidative stress resulting from free radicals hyperproduction induced by iron supplementation and harmful maternal/perinatal conditions this is rarely questioned by searchers in Africa. Success of iron supplementation has so far been assessed in terms of rise in hemoglobin level, ignoring it could be just a face of the medal.

2) If findings of this study are consistent with such a link it could lead to definition of new considerations for iron supplementation during pregnancy, mostly in conditions where deficient iron absorption precedes pregnancy.

3) The prospective nature of the study makes it possible to record data as they are generated.

\subsection{Limitations}

The major weaknesses of our study are: 1) it will not be possible to state the true onset of anemia; 2) influence and confounding effect of treatment on the course of anemia and pregnancy will be difficult to evaluate; 3) many pregnant women without obvious anemia will also be supplemented by iron, thus obscuring the causal effect of iron status on outcomes; 4) the small study sample (owed to financial constraints) will not allow us to discriminate complications according to various durations of anemia, since termination of pregnancy will occur at different gestation ages.

\section{Conclusion}

Study on potential risk of oral iron supplementation during pregnancy is op- 
portunity to fill lack of studies that have investigated possible associations, in part between unabsorbed iron and oxidative stress, and in another part between oxidative stress induced by this unabsorbed iron and adverse obstetrical outcomes.

\section{Author's Contributions}

MMA is the principal investigator, participated in designing the study and is actively involved in data collection. TUB generated and designed the study. MMR participated in designing the study. All authors contributed in drafting the manuscript.

\section{Disclosure}

The authors report no conflict of interest in this work.

\section{References}

[1] Pang, W.W. and Schrier, S.L. (2012) Anemia in the Elderly. Current Opinion in Hematology, 19, 133-140. https://doi.org/10.1097/MOH.0b013e3283522471

[2] Westerlind, B., Östgren, C.J., Mölstad, S. and Midlöv, P. (2016) Prevalence and Predictive Importance of Anemia in Swedish Nursing Home Residents-A Longitudinal Study. BMC Geriatrics, 16, 206. https://doi.org/10.1186/s12877-016-0375-2

[3] Mangla, M. and Singla, D. (2016) Prevalence of Anemia among Pregnant Women in Rural India: A Longitudinal Observational Study. International Journal of Reproduction, Contraception, Obstetrics and Gynecology, 5, 3500-3505.

[4] Tandu-Umba, B. and Mbangama, M.A. (2015) Association of Maternal Anemia with Other Risk Factors in Occurrence of Great Obstetrical Syndromes at University Clinics, Kinshasa, DR Congo. BMC Pregnancy and Childbirth, 15, 183. https://doi.org/10.1186/s12884-015-0623-z

[5] Patel, K.V. (2008) Epidemiology of Anemia in Older Adults. Seminars in Hematology, 45, 210-217. https://doi.org/10.1053/j.seminhematol.2008.06.006

[6] International Anemia Consultative Group (INACG) (2002) Why Is Iron Important and What to Do about It: A New Perspective. Report of the 2001 INACG Symposium, Hanoi, Vietnam, 15-16 February 2002, 1-50.

[7] World Health Organization (2006) Integrated Management of Pregnancy and Childbirth (IMPAC) Vol. 1.8. World Health Organization. Department of Making Pregnancy Safer (MPS); Geneva, Switzerland. Iron and Folate Supplementation. Standards for Maternal and Neonatal Care, 1-6.

[8] EFSA NDA Panel (EFSA Panel on Dietetic Products, Nutrition and Allergies) (2015) Scientific Opinion on Dietary Reference Values for iron. EFSA Journal, 13, 115. https://doi.org/10.2903/j.efsa.2015.4254 http://www.efsa.europa.eu/en/publications

[9] Paquet, I. (2011) Formation continue-Pour une santé de fer-La nutrition active pour prévenir et traiter l'anémie par déficience en fer. L'actualité pharmaceutique, 19, 1-4. http://www.professionsante.ca/files/2011/03/LPharmMar2011.pdf

[10] Schrier, S.L. and Auerbach, M. (2013) Treatment of the Adult with Iron Deficiency Anemia (à jour au 25 mars 2013). http://www.uptodate.com

[11] Association des pharmaciens du Canada (2013) Monographie des préparations 
orales de fer. Compendium des produits et spécialités pharmaceutiques. Ottawa, version en ligne e-CPS.

[12] Pharmacist's Letter (2008) Comparison of Oral Iron Supplements. Prescriber's Letter, 24, 1-4.

[13] Björn-Rasmussen, E. (1974) Iron Absorption from Wheat Bread: Influence of Various Amounts of Bran. Nutrition \& Metabolism, 16, 101-110. https://doi.org/10.1159/000175478

[14] Callender, S.T., Marney Jr., S.R. and Warner, G.T. (1970) Eggs and Iron Absorption. British Journal of Haematology, 19, 657-663. https://doi.org/10.1111/j.1365-2141.1970.tb07010.x

[15] Disler, P.B., Lynch, S.R., Charlton, R.W., Torrance, J.D., Bothwell, T.H., Walker, R.B. and Mayet, F. (1975) The Effect of Tea on Iron Absorption. Gut, 16, 193-200. https://doi.org/10.1136/gut.16.3.193

[16] Inder, T.E., Clemett, R.S., Austin, N.C., Graham, P. and Darlow, B.A. (1997) High Iron Status in Very Low Birth Weight Infants Is Associated with an Increased Risk of Retinopathy of Prematurity. Journal of Pediatrics, 131, 541-544. https://doi.org/10.1016/S0022-3476(97)70058-1

[17] Hirano, K., Morinobu, T., Kim, H., Hiroi, M., Ban, R., Ogawa, S., Ogihara, H., Tamai, H. and Ogihara, T. (2001) Blood Transfusion Increases Radical Promoting Non-Transferrin Bound Iron in Preterm Infants. Archives of Disease in Childhood. Fetal and Neonatal Edition, 84, F188-F193. https://doi.org/10.1136/fn.84.3.F188

[18] Pollak, A., Hayde, M., Hayn, M., Herkner, K., Lombard, K.A., Lubec, G., Weninger, M. and Widness, J.A. (2001) Effect of Intravenous Iron Supplementation on Erythropoiesis in Erythropoietin-Treated Premature Infants. Pediatrics, 107, 78-85. https://doi.org/10.1542/peds.107.1.78

[19] Ohlsson, A. and Aher, S.M. (2006) Early Erythropoietin for Preventing Red Blood Cell Transfusion in Preterm and/or Low Birth Weight Infants. The Cochrane Database of Systematic Reviews, 19, CD004863. https://doi.org/10.1002/14651858.CD004863.pub2

[20] Favier, A. (2003) Le stress oxydant: Intérêt conceptuel et expérimental dans la compréhension des mécanismes des maladies et potentiel thérapeutique. L'actualité chimique, 108-115.

[21] Miller, C.J., Rose, A.L. and Waite, T.D. (2016) Importance of Iron Complexation for Fenton-Mediated Hydroxyl Radical Production at Circumneutral pH. Front in Marine Science, 3,134. https://doi.org/10.3389/fmars.2016.00134

[22] Cadenas, E. (1989) Biochemistry of Oxygen Toxicity. Annual Review of Biochemistry, 58, 79-110. https://doi.org/10.1146/annurev.bi.58.070189.000455

[23] Fulbert, J.C. and Cals, M.J. (1992) Les Radicaux libres en biologie clinique. Pathologie Et Biologie, 49, 66-77.

[24] Nadal, M., Le Guyader, N., Arlet, J.B. and Husson, M.C. (2009) Chélateurs du fer et hémosidérose post-transfusionnelle. Dossier du CNHIM, Tome XXX, 4, 11-17.

[25] Zaretsky, M.V., Alexander, J.M., Byrd, W. and Bawdon, R.E. (2004) Transfer of Inflammatory Cytokines across the Placenta. Obstetrics \& Gynecology, 103, 546-550. https://doi.org/10.1097/01.AOG.0000114980.40445.83

[26] Gupta, S., Agarwal, A., Banerjee, J. and Alvarez, J.G. (2007) The Role of Oxidative Stress in Spontaneous Abortion and Recurrent Pregnancy Loss: A Systematic Review. Obstetrical \& Gynecological Survey, 62, 335-347.

https://doi.org/10.1097/01.ogx.0000261644.89300.df 
[27] Class, Q.A., Lichtenstein, P., Longstrom, N. and D’Onofrio, B.M. (2011) Timing of Prenatal Maternal Exposure to Severe Life Events and Adverse Pregnancy Outcomes: A Population Study of 2.6 Million Pregnancies. Psychosomatic Medicine, 73, 234-241. https://doi.org/10.1097/PSY.0b013e31820a62ce

[28] Dole, N., Savitz, D.A., Hertz-Picciotto, I., Siega-Riz, A.M., McMahon, M.J. and Buekens, P. (2003) Maternal Stress and Preterm Birth. American Journal of Epidemiology, 157, 14-24. https://doi.org/10.1093/aje/kwf176

[29] Grote, N.K., Bridge, J.A., Gavin, A.R., Melville, J.L., Iyengar, S. and Katon, W.J. (2010) A Meta-Analysis of Depression during Pregnancy and the Risk of Preterm Birth, Low Birth Weight, and Intrauterine Growth Restriction. Arch Gen Psychiatry, 67, 1012-1024. https://doi.org/10.1001/archgenpsychiatry.2010.111

[30] Khasan, A.S., McNamee, R., Abel, K.M., Pedersen, M.G., Webb, R.T., Kenny, L.C., Mortensen, P.B. and Baker, N.P. (2008) Reduced Infant Birth Weight Consequent upon Maternal Exposure to Severe Life Events. Psychosomatic Medicine, 70, 688-694. https://doi.org/10.1097/PSY.0b013e318177940d

[31] Tegethoff, M., Greene, N., Olsen, J., Meyer, A.H. and Meinlschmidt, G. (2010) Maternal Psychosocial Adversity during Pregnancy Is Associated with Length of Gestation and Offspring Size at Birth: Evidence from a Population-Based Cohort Study. Psychosomatic Medicine, 72, 419-426. https://doi.org/10.1097/PSY.0b013e3181d2f0b0

[32] Latendresse, G., Ruiz, R.J. and Wong, B. (2013) Psychological Distress and SSRI Use Predict Variation in Inflammatory Cytokines during Pregnancy. Open Journal of Obstetrics and Gynecology, 3, 184-191. https://doi.org/10.4236/ojog.2013.31A034

[33] Lee, S.Y., Park, K.H., Jeong, E.H., Oh, K.J., Ryu, A. and Park, K.U. (2012) Relationship between Maternal Serum C-Reactive Protein, Funisitis and Early-Onset Neonatal Sepsis. Journal of Korean Medical Sciences, 27, 674-680. https://doi.org/10.3346/jkms.2012.27.6.674

[34] Sorokin, Y., Romero, R., Mele, L., Wapner, R.J., Iams, J.D., et al. (2010) Maternal Serum Interleukin-6, C-Reactive Protein, and Matrix Metalloproteinase-9 Concentrations as Risk Factors for Preterm Birth $<32$ Weeks and Adverse Neonatal Outcomes. American Journal of Perinatology, 27, 631-640. https://doi.org/10.1055/s-0030-1249366

[35] Sykes, L., MacIntyre, D.A., Yap, X.J., Teoh, T.G. and Bennett, P.R. (2012) The Th1:Th2 Dichotomy of Pregnancy and Preterm Labour. Mediators of Inflammation, 2012, Article ID: 967629. https://doi.org/10.1155/2012/967629

[36] Walsh, S.W. (1998) Maternal-Placental Interactions of Oxidative Stress and Antioxidants in Preeclampsia. Seminars in Reproductive Endocrinology, 16, 93-104. https://doi.org/10.1055/s-2007-1016256

[37] Morris, J.M., Gopaul, N.K., Enderesen, M.J., Knight, M., Linton, E.A., Dhir, S., Anggard, E.E. and Redman, C.W. (1998) Circulating Markers of Oxidative Stress Are Raised in Normal Pregnancy and Pre-Eclampsia. British Journal of Obstetrics and Gynaecology, 105, 1195-1199. https://doi.org/10.1111/j.1471-0528.1998.tb09974.x

[38] Mutlu-Turkoglu, U., Ademoglu, E., Ibrahimoglu, L., Aykac-Toker, G. and Uysal, M. (1998) Imbalance between Lipid Peroxidation and Antioxidant Status in Preeclampsia. Gynecologic and Obstetric Investigation, 46, 37-40. https://doi.org/10.1159/000009994

[39] Shaarawy, M., Aref, A., Salem, M.E. and Sheiba, M. (1998) Radical-Scavenging Antioxidants in Pre-Eclampsia and Eclampsia. International Journal of Gynecology \& 
Obstetrics, 60, 123-128. https://doi.org/10.1016/S0020-7292(97)00256-7

[40] Poranen, A.K., Eklad, U., Uotila, P. and Ahotupa, M. (1996) Lipid Peroxidation and Antioxidants in Normal and Pre-Eclampsia Pregnancies. Placenta, 17, 401-405. https://doi.org/10.1016/S0143-4004(96)90021-1

[41] Stein, P., Scholl, T.O., Schluter, M.D., Leskiw M.J., Chen, X., Spur, B.W. and Rodriguez, A. (2008) Oxidative Stress Early in Pregnancy and Pregnancy Outcome. Free Radical Research, 42, 841-848. https://doi.org/10.1080/10715760802510069

[42] Zein, S., Rachidi, S. and Hininger-Favier, I. (2013) Fer, stress oxydant et diabète gestationnel. Médecine des Maladies Métaboliques, 7, 144-148. https://doi.org/10.1016/S1957-2557(13)70511-X

[43] Leverve, X. (2003) Hyperglycemia and Oxidative Stress: Complex Relationships with Attractive Prospects. Intensive Care Medicine, 29, 511-514. https://doi.org/10.1007/s00134-002-1629-3

[44] Berger, M.M. (2006) Nutritional Manipulation of Oxidative Stress: Review of the Evidence. Nutition Clinique et Métabolisme, 20, 48-53. https://doi.org/10.1016/j.nupar.2005.12.005

[45] Kasiulevičius, V., Šapoka, V. and Filipavičiūtè, R. (2006) Sample Size Calculation in Epidemiological Studies. Gerontologija, 7, 225-231.

[46] World Health Organization (2001) Iron Deficiency Anaemia: Assessment, Prevention, and Control. WHO/NHD, Genève. http://www.who.int/iris/bitstream/10665/66914/1/WHO_NHD_01.3.pdf?ua=1

[47] World Health Organization (2008) Worldwide prévalence of anaemia 1993-2005, WHO Global Database on Anaemia. http://www.who.int/vmnis/publications/anaemia_prevalence/en/index.html

[48] Berger, J. and Dillon, J.C. (2002) Stratégies de contrôle de la carence en fer dans les pays en développement. Cahiers Santé, 12, 22-30.

[49] Joshi, S.R., Mehendale, S.S., Dangat, K.D., Kilari, A.S., Yadav, H.R. and Taralekar, V.S. (2008) High Maternal Plasma Antioxidant Concentrations Associated with Preterm Delivery. Annals of Nutrition and Metabolism, 53, 276-282. https://doi.org/10.1159/000189789 